Please do not remove this page

RMIT

UNIVERSITY

\title{
Analyzing geographic query reformulation: an exploratory study
}

Aloteibi, Saad; Sanderson, Mark

https://researchrepository.rmit.edu.au/esploro/outputs/9921859835401341/filesAndLinks?institution=61RMIT_INST\&index=null

Aloteibi, S., \& Sanderson, M. (2014). Analyzing geographic query reformulation: an exploratory study. Journal of the Association for Information Science and Technology, 65(1), 13-24.

https://doi.org/10.1002/asi.22961/pdf

Document Version: Accepted Manuscript

Published Version: https://doi.org/10.1002/asi.22961/pdf

Repository homepage: https://researchrepository.rmit.edu.au

(c) 2013 ASIS\&T

Downloaded On 2023/04/26 23:59:29 +1000

Please do not remove this page 
Thank you for downloading this document from the RMIT Research Repository.

The RMIT Research Repository is an open access database showcasing the research outputs of RMIT University researchers.

RMIT Research Repository: http://researchbank.rmit.edu.au/

\section{Citation:}

Aloteibi, S and Sanderson, M 2014, 'Analyzing geographic query reformulation: an exploratory study', Journal of the Association for Information Science and Technology, vol. 65, no. 1, pp. 13-24.

See this record in the RMIT Research Repository at:

https://researchbank.rmit.edu.au/view/rmit:26920

Version: Accepted Manuscript

Copyright Statement:

(c) 2013 ASIS\&T

Link to Published Version:

http://dx.doi.org/10.1002/asi.22961.pdf 


\title{
Analyzing Geographic Query Reformulation: An Exploratory Study
}

\author{
Saad Aloteibi \\ University of Cambridge, The Computer Laboratory, Cambridge, United Kingdom \\ Tel: +441223763528 \\ Fax: +441223334678 \\ Email: Saad.Aloteibi@cl.cam.ac.uk \\ Mark Sanderson (corresponding author) \\ RMIT University, School of Computer Science and Information Technology, \\ Melbourne, Victoria, Australia \\ Tel: +61399259675 \\ Fax: +61399253748 \\ Email: mark.sanderson@rmit.edu.au
}




\title{
Analyzing Geographic Query Reformulation: An Exploratory Study
}

\begin{abstract}
Search engine users typically engage in multi-query sessions in their quest to fulfill their information needs. Despite a plethora of research findings suggesting that a significant group of users look for information within a specific geographical scope, existing reformulation studies lack a focused analytical study of how users reformulate geographic queries. This study aims to comprehensively investigate the ways in which users reformulate such needs in an attempt to fill this gap in the literature. Reformulated sessions were sampled from a query log of a major search engine to extract 2,400 entries that were manually inspected to filter geo-sessions. This resulted in identifying 471 search sessions to have geographical intent and were analyzed quantitatively and qualitatively. The results revealed that one in five of the users who reformulated their queries were looking for geographically related information. They mostly reformulated their queries by changing the content of the query rather than the structure. Users were not following a unified sequence of modifications and mostly performing a single reformulation action. However, in some cases it was possible to anticipate their next move. A number of tasks in geo-modifications were identified including: standard, multi-needs, multi-places, and hybrid approaches. The research concludes that it is important to specialize query reformulation studies to focus on particular query types rather than generically analyzing them as it is apparent that geographic queries have their special reformulation characteristics.
\end{abstract}

\section{Introduction}

Information retrieval research has a long standing focus on analyzing the ways by which information seekers express and re-express their needs when interacting with an IR system. One of the most popular IR systems is the search engine, which has been the main focus of such studies. Earlier work such as Silverstein et al.'s (1998) study aimed at understanding the language by which users formulate their needs generically. Subsequent studies as in (Bruza \& Dennis, 1997; Lau \& Horvitz, 1999; Spink et al., 2000; Rieh \& Xie, 2006; Liu \& Belkin, 2008; Jansen et al., 2009a) were motivated by the fact that users tend to interact with the IR system by reformulating their queries in order to satisfy an information need. Although it is apparent that analyzing reformulated queries will be a closer proxy to understanding users' needs and how they express them in comparison with queries taken out of their context; little of this work, however, has focused on particular query types. One specific type of interest is geographic queries, which have been found to account for a significant proportion of web searching (Sanderson \& Kohler, 2004; Jones et al., 2008).

One exception is the study conducted by Jones et al. (2008) who considered modifications of such queries by automatically examining the geo and the nongeo parts of the query. Unlike Jones et al.'s study who focused on proximity analysis of users' location and their sought-after geographically constrained 
information needs, we take a qualitative approach towards analyzing geosessions by comprehensively characterizing reformulation patterns and search tasks. Unlike statistical approaches and unsupervised clustering, qualitative analysis excels in uncovering new patterns in the dataset that are beyond statistical methods. For example, the decision of whether to classify a certain query as specialization or generalization of another is a non-trivial decision that will essentially require manually labeled data to train a model. Also uncovering prominent search tasks such as "one-place multiple needs" in geo-sessions requires a manual study of such sessions which previous automatic methods such as the ones in Jones et al. (2008) cannot uncover. Moreover, a qualitative understanding of geographically related queries, which our results contribute to, have the potential to guide local search and suggestion models. For example, we show that about $30 \%$ of geographic sessions are not centered on a single information need; suggesting that specialized diversification methods would benefit from balancing between the three main tasks identified in this study that are likely to appear in geographically related queries. In addition, we show that users persist in modifying a specific part of the query (e.g. the geo or the content component). This is a useful signal to contextualize query suggestions and for advertisements selection. To obtain such results, we take a data driven qualitative approach by analyzing a query log of a major search engine company. The aims of the work were set out as follows.

1. To identify the size of query reformulation.

2. To examine the significance of geographic reformulation in web searching.

3. To examine the difference between geographic and non-geographic searching sessions.

4. To examine the nature of geo-query reformulation.

5. To examine actions in geo-reformulation.

6. To explore geo-reformulation tasks.

The remainder of this paper presents a review of past work, followed by details of the research framework and methods. Next, the study findings are described and their value detailed. Finally conclusions are drawn and possible directions for future work are outlined.

\section{Literature review}

Query Logs Analysis (QLA) studies can be classified into two main research streams: informative and supportive. The former tend to describe how users are searching the web, what they are looking for, and why they search; the latter treat query logs as a resource that can be used directly to enhance the performance of services, such as query suggestion (Cucerzan \& White, 2007), query expansion (Cui et al., 2003) and query rewriting (Jones et al., 2006). Here, we present notable studies from the informative theme; we describe studies in reformulation with more emphasis on studies covering geographical queries; and we shed light on the identification of geographical queries.

The first major informative study was conducted by Silverstein et al. (1998), who sampled about 1 billion search requests to the AltaVista search engine, from which they concluded that Internet searchers usually issue short queries (average of 2.35 terms per query) and only review the top 10 results. Similar findings were reported by (Jansen et al., 1998; Spink et al., 2001; Park et al., 
2005). Silverstein et al. (1998) found that users occasionally modify their queries, however, the absence of analyzing the click-through data in Silverstein et al.'s work made it hard to understand if the lack of modification was due to search engine success or a failed search session.

In a series of studies conducted on the Excite search engine, a wider range of observations about users' behavior was reported (Jansen et al, 1998; Jansen et al., 2000; Wolfram et al., 2001; Spink et al., 2001; Spink et al., 2002a). For instance, with the relevance feedback feature which expand users' queries given users' relevance judgment, they reported that less than $10 \%$ of the queries came from this service. Another result is that they found the query structure to be simple, rarely containing Boolean or other search operators. Zhang \& Moffat (2006) analyzed a query log containing click-through data. One of their results was that the majority of users make their reformulation decisions within seconds, suggesting that users are often not certain of the query that best represents their information need and that they have more than one formulation in mind, which they try sequentially.

Another body of research focused on groups of users based either on their nationality as in Spink et al. (2002a) for U.S. and European countries; Efthimiadis (2008) for Greek; Park et al. (2005) for Koreans and Chau et al. (2009) for Chinese. Others have analyzed query characteristics, such as in the work of Bendersky \& Croft (2009), who focused on long queries, or Spink \& Ozmultu (2002) for queries in a question format.

Another grouping criterion is based on the query topic or type which aims to discover the topics which people search for. An early study which was directed towards this goal was conducted by Ross \& Wolfram (2000) using an Excite search log. They revealed that adult-related material was the most requested topic on the web, whereas documents related to business and computing were ranked in the middle of their 30 categories list. In 2002, Spink et al. (2002b) extended the former study by comparing samples extracted from the same search engine in 1997, 1999, and 2001. They found that users' interests on the web had shifted from adult to business related topics. Specifically, they reported that "Commerce, travel, employment or economy" and "People, places or things" categories encompassed the most frequent queries. A later study by Jansen \& Spink (2006) confirmed these findings. Pass et al. (2006) used different categorization criteria to the previously mentioned studies. They reported that queries related to entertainment and shopping were among the most asked queries; computing, news, health, business, and places were of middling interest, holidays and finance were the least demanded.

Sanderson \& Kohler (2004) took a different approach by considering the geographical scope of users information needs. They investigated the commonality of geographically related queries and aimed to answer the questions of what users are looking for when submitting a geographic query and partially how they search for it. Based on a manual analysis of one of the Excite query logs, they revealed that geo-queries represent nearly one fifth of the overall queries, with $80 \%$ of those geo-queries containing a place name $(14.8 \%$ 
of all queries). Similar findings were reported in Gan et al. (2008) and Jones et al. (2008). Sanderson \& Kohler's (2004) geo-queries were found to be $25 \%$ longer than the average query with a mean of 3.3 terms. They related this result to the structure of geo-queries being composed of both geo and non-geo parts. Their results showed that users' interests when searching for geographically related information did not significantly differ from the general trend, as reported in Spink et al.'s (2002b).

Gan et al. (2008) analyzed a query log from AOL containing about 36 million queries. They found that the likelihood of tagging a query as a geo-query increased as the number of terms in the query grew. They attempted to answer the question of why users search for geo-information and reached the conclusion that "geo queries are more frequently aimed at locating goods and services" (Gan et al., 2008: 51). In addition, they stated that some of the geo-queries were navigational queries, in which the user was either trying to distinguish a site from one another by using a geo-flag, such as in "Google UK", or filtering a particular site's content according to the location of interest (e.g. "craigslist Los Angeles").

\subsection{Query Reformulation}

Query reformulation has received much attention in the QLA literature. Silverstein et al.'s (1998) study revealed that $12 \%$ of reformulated queries were a result of adding or deleting a term or a search operator to the previous query. Specifically, they stated that the addition of words was often coupled with the use of the "-" operator in an attempt to restrict the retrieved documents. Meanwhile, it was shown that over half of users reformulated their queries by using a mixture of adding and deleting words. The authors concluded that "the user is modifying the query not to change the scope of the query, but to restate the information need" (Silverstein et al., 1998: 11).

Jansen et al. (2000) explored users' reformulation behavior by examining successive queries in order to detect the changes in the number of terms. They assumed that if a follow-up query had $(n)$ number of terms more than the previous query, it represented the user adding terms and vice versa. In cases where the number of terms was identical between the two queries it was regarded as a zero change since users might add and delete terms at the same time. They found that $34.8 \%$ of queries had zero change. A similar figure was found in Spink et al. (2001). The remaining percentage was divided nearly equally between adding and deleting. This was contrary to Spink et al.'s (2001) findings that $41.6 \%$ of users modified their queries by adding terms and $25.9 \%$ by deletion. The researchers also showed that Internet searchers tend to go from general queries to more specific ones. However, it should be noted that such figures can be affected by the session identification procedure, which neither Jansen et al. (2000) nor Spink et al. (2001) provided information on. Park et al. (2005:215) found that "more users changed their queries entirely rather than adding terms to or deleting terms from the previous queries". Their work was based on a Korean search engine. 
Lau \& Horvitz (1999) focused on building probabilistic models that capture users' refinement actions. They took a broader view of reformulation from past studies and proposed seven types of reformulation: new, generalization, specialization, reformulation, interruption, request for additional results, and blank queries. These classes were assigned manually. The researchers found that users refine their queries by either asking for additional results or starting a new search as opposed to expanding or contracting a query. Spink et al. (2000) categorized reformulated queries into four groups: unique query, modified query, next page, and relevance feedback. Their results were broadly consistent with Lau \& Horvitz (1999); in addition, they examined query patterns during search sessions, from which they argued that the most common pattern of query reformulation was to type a unique query followed by viewing more results.

Rieh \& Xie (2006: 755) argued that "the interactive IR process is cyclical in the sense that both query terms and search results are constantly selected, evaluated and modified". They extended previous work (Rieh \& Xie, 2001) from which they discovered that modifications lie in three main areas: content in which the user changes the query meaning; format when the structure of the query is modified but not the meaning; and resource when choosing between different document types. These three classes were then divided into sub-classes in order to capture more specific reformulation patterns. Although their study provides an in-depth analysis of query modification, it might be limited with the fact that they defined a reformulated session as having six or more queries which seems to be relatively high and thus they might have ignored some patterns which were more common in shorter sessions.

Jansen et al. (2009a) studied query modification from a different perspective. They utilized the concept of a state transition map to infer when users were most likely to seek help and to which reformulation state they will move. Their research was motivated by the fact that contextualizing query suggestions would reduce the cognitive overhead that is required to process the additional information offered to users.

\subsection{Query Reformulation from a geographical perspective}

In her geo-query research, Kohler (2003) described query reformulation behavior based on sessions that contained the place name "London". Her analysis of the addition/deletion of terms in successive queries did not significantly differ from that of Spink et al. (2001). Moreover, it was noticed that the addition of more geo terms in the query was commonly used to differentiate between places that shared the same name. Unlike Kohler (2003), this study is not limited to a single location but rather aims to draw global conclusions. Gan et al. (2008) briefly covered geo-modification and argue that reformulation was not common in web searching and the non-geo part of the query is the most likely component to be reformulated.

Jones et al. (2008) appears to be the first to examine geo-modification as a main research problem. Their study utilized a corpus of query logs extracted from Yahoo search engine. Their main goal was to examine geographical proximity between the searcher location and her intended geographical need. This relied 
on using the current user IP address and calculating the distance between the IP address and the geographical entity in the query. This approximation of distance revealed some interesting patterns. They showed that users tend to search for "maps" for locations that are far from their current location suggesting unfamiliarity with the sought-after place while they tend to search for local services such as "restaurants" and "schools" near to their current location. They then applied the same procedure to pair of reformulated queries and found that users tend to not expand their geographical scope to a further place but remain within the initial scope. While it is useful to understand users' perceived sense of proximity in regard to geographically constrained queries by using such global statistics as a proxy, it is also important to understand what prompt a geographically related information need spanning a search session of more than a pair of queries in the first place. Also and in contrast to their analysis, we employ the search session as the unit of analysis in addition to pairs of subsequent geographical queries.

In addition to their proximity analysis, Jones et al. (2008) applied a chain of filters to extract 108 geographical queries which were then classified into two broad categories, namely reformulation that includes changing the geo or the non-geo part of the query; spell correction or place disambiguation. They found that 86 of their sample queries belong to the reformulation category while 12 were classified under the second category. The remaining queries were considered to be false positive. However, it is hard to draw conclusions from such small study sample and from such coarse-grained classification. In addition, they also evaluated an automated reformulation tool that uses query logs as a source of candidate words (Jones et al., 2006) in terms of the suggestion of georewrites and found that its result was deemed to be relevant when the geo element of the query was unchanged compared with its former.

\subsection{The identification of geographic queries}

Since most of the transaction logs available are for generic search engines, an initial stage of research in this field was to define and extract those queries that had a geo-intent. Different approaches to tackle this were proposed. Kohler (2003) simply inspected queries manually, looking for terms that were geographically inductive, such as place names, adjective of places and place locators.

Jones et al. (2008) devised an automated method that assigned a score to each term in the query as well as a "location-related probability" based on a geographic database. The final judgment as to whether a query was geo or not was affected by the former values combined with values from another database of context words and non-place names (e.g. "George Washington"). When a query was tagged as geo, the researchers checked the user IP address and the frequency of occurrence for that particular place in order to identify the intended location in cases when many geographic entities existed with the same name. This approach might decrease the probability of false positive identification, however, it is used only for "place names" detection and not geographic query identification. This is because less than $50 \%$ of geo-queries contain a place name (Welch \& Cho, 2008; see also Yi et al. 2009). For such 
implicit geo-references queries, Martins et al. (2006) mined the geographic scope of the retrieved documents that matched the query. Wang et al. (2005: 424) coined the term "Query Dominant Location", which they defined as "the geographical location(s) associated with a query in collective human knowledge". Wang et al. (2005) also attempt to identify the implicit geo-queries by mining the top retrieved documents.

In contrast to such approaches, we have conducted a manual annotation of a statistically representative sample in order to overcome polysemy and ambiguity issues in search queries. Such problems are likely to have affected previous methods which rely on dictionary-based classification. In addition, characterizing geo-sessions in terms of modification patterns and search task is inherently a semantic task that needs to incorporate further reasoning about various contextual signals such as previous queries in the session; semantic and lexical relations between successive queries; clicked documents and the induction of search tasks. In conclusion, it is clear that geographic queries are common, yet the study of them, particularly in sessions is not well understood. The following section addresses the methodology for our study of geographic query sessions.

\section{Methodology}

In this section we provide a detailed description of the data used in the experiments along with the design of the analysis.

\subsection{Definition of terminology}

Jansen \& Pooch (2001) highlighted the importance of defining the terminology used in QLA studies in order to pave the way for valid comparison between different studies. Hence, we derive our terminology from previous research in the field where ever possible. As described in the following section, the data we are working with is a query log from Microsoft Live search engine, and our terminology is as follows:

- Term: "A term is any unbroken string of characters (i.e. a series of characters with no space between any of the characters)" (Jansen et al., 2000: 212). Thus in this research, search operators were dealt with as terms.

- Query: "A query consists of one or more search terms" (Jansen et al., 2000: 212).

- Session: The Microsoft log has session identifiers determined by a time cut of twenty minutes; if the user status was idle for longer, the session was deemed finished. Thus, we could define a session for the purpose of this study as "a series of queries submitted within a time-frame of 20 minutes between a query and its subsequent query". However, it is worth noting that the session mark-up procedure appears imperfect as we identified a small number of sessions longer than 20 minutes.

- Initial query: "The first query in a session" (Jansen \& Pooch, 2001: 244).

- Reformulated query: "A subsequent query in a session that is different than any of the previous queries" (Jansen \& Pooch, 2001: 244). Such a difference between queries occurred when the user restated his/her request in several ways but the underlying information need was unchanged. 
- Repeated query: A subsequent query that was identical to a former one. These queries were ignored since there are at least two interpretations for this type: either the user was rewriting the exact query again or reviewing the next pages on the result page (Jansen et al., 2000). The log we have did not allow us to judge between these two activities and, therefore, it was excluded from our analysis.

- Geographic query: "A search query with a geographic intent". This intent could be explicitly pronounced as part of the query by the inclusion of geographical entity such as city names or places or implicitly pronounced (Wang et al., 2005).

\subsection{Research Design}

A query log of Microsoft Live search engine was made available to this study under the "Microsoft live lab: Accelerating Search in Academic Research" plan in 2006. The data, known as "Microsoft 2006 RFP", was collected over one month, starting from $1 / 5 / 2006$ to $31 / 5 / 2006$. It was released to participants of the "Workshop on Web Search Click Data" hosted at the WSDM conference in 2009. Despite its relative age, it is the only large scale data set available to academic groups. It is still actively used in research published in major research forums: Broccolo et al (2012); Bendersky et al (2011); Zhu et al (2011).

It contained around 14,921,285 queries encompassing 7,470,915 sessions from US users with a click stream of 12,251,067 records. For each query, the dataset contains information about the time it was submitted; the search session it belongs to; number of results shown in the result page; and the documents' URLs that the user clicked on and their position in the result page if any.

\subsubsection{Data preparation}

Before analyzing a query log it is necessary to consider queries submitted by bots or online monitoring systems that routinely queries the web through the search engine API to collect results with respect to predefined template of queries. Silverstein et al. (1998) did not remove such queries; as a result, they reported a large standard deviation in regard to the number of queries per session (123.40). Such session tend to have anomalous characteristics such as an excessive number of reputations that would clearly bias the derived statistics. Moreover, since we were only concerned with human interaction with the search engine, we filtered non-human queries from the logs. Jansen (2009b) suggested cleaning all the sessions that have a length of over 100 queries, which is the approach we adopted. Although this is not a perfect method, it does reduce the effect of noisy data. After applying this filtering approach, we removed 110,296 queries that amounts for 305 sessions. The resulted standard deviation was reduced from 12.52 to 2.24 .

Note, before releasing their log data, Microsoft removed e-mail addresses and sequences of numbers longer than 8 digits in the queries replacing them with special characters. IP addresses were anonymized, which limited this study in that we could not identify the distance between the user location and his/her geographic query. 


\subsubsection{Trying to identify geographic queries automatically}

An attempt to automatically extract geographic queries was made adopting Martins et al.'s (2006) triple <what, relation, where>. We used a database containing 1,884,611 geographic features in the US, taken from the Geonames server, and a defined set of 73 spatial terms, for example "in", "near" and "north". Firstly, we filtered the data to extract queries that contained a spatial term, and then we filtered the result against the geographic references database as an application of Martins et al.'s method. However, this approach introduced a high false positive rate to our potential geo-dataset. Therefore, we choose not to proceed with this approach, instead using a manual identification approach based on a sample of the log.

\subsubsection{Sampling the data for manual analysis}

To represent a thorough consideration of geo-modification, we extracted those queries that were reformulated. Rieh \& Xie (2006) decided to study sessions that had over five queries; Spink et al. (2000) qualitatively analyzed 191 sessions that had two or more queries; Jones et al. (2008), who have studied georeformulation, labeled 108 queries which passed four different filtering algorithms. In contrast to the previous studies, our approach was as follows. We filtered the raw dataset to extract all sessions that had at least three queries. The intent behind this was to include the sessions where users persevere in modifying their queries as this might give an insight into the cognitive processes followed by users. This resulted in a new dataset, which we refer to as "reformulated queries". It had 1.5 million sessions, composed of 7.5 million queries. The following formula showed that a sample size of 2,400 queries was needed given required confidence levels, error rates and time available.

$$
\begin{gathered}
\text { Initial Sample Size }=\frac{Z^{2} * P *(1-P)}{C^{2}} ; \\
\text { Final Sample Size }=\frac{\text { Initial Sample Size }}{1+\frac{\text { Initial Sample Size }-1}{\text { Population }}}
\end{gathered}
$$

Where:

- $\mathrm{Z}$ is the confidence level, chosen to be $95 \%$.

- $\mathrm{P}$ is the response distribution; by default it is 0.5 .

- $\mathrm{C}$ is the confidence interval (i.e. the error rate), chosen to be $2 \%$.

- Population is our reformulated queries; its value is 7,511,984

Thus, we sampled 2,400 queries out of the 7.5 million queries. Each query was then subject to a manual binary classification as a query with geographical intent or not. In cases where the intent was not easily identifiable by looking at the query itself, we retrieved all the queries that occur in the same session as the query in question. We then used this information to further investigate whether the query has a geographical intent or not. 


\subsection{Data Analysis}

Here we describe the data preparation needed to meet our research objectives.

Objective 1: Identifying the size of query reformulation: by macro-analyzing the raw dataset looking for the number of queries per session, we were able to draw a conclusion of the distribution of session lengths.

Objective 2: Examining the significance of geographic reformulation in web searching: as our focus was on reformulated queries, we checked if the session contains a geo-part in any of its queries. If not, the session was excluded from this analysis. We kept the sessions of the identified geographic queries for the purpose of analyzing them. For example, the query "Regency inn" implies an implicit geographical query. By inspecting the session history for this query, we found that the user was looking for this hotel in the city of Sewanee in Tennessee State. Other examples like "iad" or "Helen bernhart bakery" refers to geographical entities by the use of acronyms or physical entities references such as "bakery". These queries were considered to have a geographical intent.

Objective 3: Examining the difference between geographic and nongeographic searching sessions: we compared the length of geo-sessions identified in objective 2 with the length of non-geo sessions. Applying the t-test we were able to see whether there was a difference between the two or not.

Objective 4: Examining the nature of geo-query reformulation: we applied Spink et al.'s (2000) method by considering the following: If the successive query had the same number of words then the change was deemed to be ( 0 changes). If the successive query had $n$ words more than its former then the change was deemed to be a $+n$ change and vice versa. However, a reformulation process can be interrupted by navigational queries (Broder, 2002; Rose \& Levinson, 2004). Including such queries, risk skewing results, thus, it was decided to exclude navigational queries from reformulations.

Objective 5: Examining actions in geo-reformulation: studies in query reformulation have usually dealt with this issue by proposing a number of modification actions that capture the different ways in which searchers reformulate their needs (Rieh \& Xie, 2006; Spink et al., 2000; Jones et al., 2008; Bruza \& Dennis, 1997; Lau \& Horvitz, 1999). We adopt Rieh \& Xie's (2006) classification taking into account the consensus in the literature that a geo-query has two elements: the object which searchers are looking for and the location in which they are interested (Gan et al., 2008; Martins et al., 2006). By doing this, we were able to examine whether users often reformulate the geo or the non-geo component. The following shows the classification used in this study from three levels. The reason for having these levels is twofold: to represent geomodification from different levels of abstraction; and to avoid the "long tail" effect by having many specific categories. Table 1 illustrates this idea:

It should be noted that some of the queries might have two reformulation actions since the user, for example, may generalize the geographic location and specialize the non-geo. Therefore, we assigned a weight for each action 
depending on the total number of changes that the user had made to the query according to the following formula:

$$
\text { Action Weight }=\frac{1}{\text { the total number of changes performed on the query }}
$$

When designing the transition matrix, we took into consideration the current state of the query, that is the reformulation action that had been performed on the query, and the next state the user had moved to. We assumed that the next state is only dependent on the current modification state. This approach was followed in Pass et al. (2006) and Spink et al. (2000).

\begin{tabular}{lll} 
No. & Query & State \\
\hline 1 & Gas Motor Bicycles & Initial query \\
2 & Gas Bicycles & Non-geo Generalization \\
3 & Gas Bicycles Los Angeles & Geo Specialization \\
Table 1: Illustration of geo-reformulation mark up &
\end{tabular}

Objective 6: Exploring geo-reformulation tasks: Rieh \& Xie (2006) argue that a major limitation of research on query reformulation is the consideration of the changes in successive queries only without inferring the whole process. We qualitatively analyzed the geo-sessions looking for particular trends that could be descriptive of the search task.

\section{Results}

This section details the results of the research along with their implications and position with the existing literature. It is structured as follows: firstly, the results of analyzing the dataset as a whole are reported in order to examine the length of searching sessions; secondly, the findings of the sampled data analysis are presented. Note that all reported percentages are taken from a sample the size of which means one can be $95 \%$ confident that the true value lies within an interval of $\pm 2 \%$ of the reported value. Results $<2 \%$ are judged to be too small to be confident about and are not reported.

\subsection{Identifying the size of query reformulation}

The general consensus in past work is on the shortness of searching sessions. Our results concur with this since we found that $62.1 \%$ of sessions contained one query, and $17.8 \%$ had two queries. The mean session length before and after filtering the data from agent queries was unchanged: 1.99 and 1.98 , respectively; similar to Silverstein et al.'s (1998) mean length of 2.02; somewhat larger than Zhang \& Moffat's (2006) figure of 1.55. It should be noted that their filtering approach to clean queries was more aggressive than ours or Silverstein's. We further examined the click data of the single query sessions: $69.6 \%$ viewed a result. The user logs do not record any user action for the remaining 30.4\% of sessions. 


\subsection{Geographic reformulation on the web}

The analysis of the 2,400 reformulated queries revealed that $471(19.6 \%)$ have a geographical intent. Given the diversity of topics searched for on the web and that our data were extracted from a general purpose search engine, such a figure is striking, indicating much geographic interest when searching the web and a persistent tendency to include a geographic entity while searching taking into account Sanderson \& Kohler's (2004) earlier analysis of the older Excite query logs. The mean length of geo-sessions was 7.6, similar to the non-geo sessions figure of 7.5 queries per session. The difference between the geo-sessions and the non-geo sessions in terms of their length per query was not statistically significant when applying the t-test. In the following, we investigate different aspects of reformulations in geographical sessions.

\subsection{The nature of geo-query reformulation}

We enriched the 471 queries sample with all the queries that occurred within the same session as the sampled query, which resulted in a total of 2,458 queries. These 471 geo-sessions were analyzed to shed light on the difference between a pair of geographic queries in terms of the query length. Table 2 shows the result obtained.

\begin{tabular}{rrrrrrrrr}
\multicolumn{3}{c}{ Adding words } & \multicolumn{3}{c}{ Deleting words } & \multicolumn{3}{c}{ Substituting words } \\
Words & Freq. & \% & Words & Freq. & \% & Words & Freq. & \% \\
\hline 1 & 427 & $21.5 \%$ & -1 & 316 & $15.9 \%$ & 0 & 588 & $29.6 \%$ \\
2 & 231 & $11.6 \%$ & -2 & 190 & $9.6 \%$ & & & \\
3 & 76 & $3.8 \%$ & -3 & 67 & $3.4 \%$ & & & \\
$4-8$ & 43 & $2.2 \%$ & $-4--8$ & 49 & $2.5 \%$ & & & \\
Total & $\mathbf{7 7 7}$ & $\mathbf{3 9 . 1 \%}$ & Total & $\mathbf{6 2 2}$ & $\mathbf{3 1 . 3 \%}$ & Total & $\mathbf{5 8 8}$ & $\mathbf{2 9 . 6 \%}$ \\
Table 2: Geo-modification - changes in successive query length & & & &
\end{tabular}

It is evident that word substitution is the most frequent single reformulation pattern, with a proportion of 588 occurrences among all 1987 reformulated queries. These figures are consistent with the generic analysis published in Spink et al. (2000). From a developer's point of view, such figures might raise the need for modifying the way search engines propose reformulated queries to balance and pay attention to word substitution. This claim could support the work of Jones et al. (2006), who proposed a similar method. However, from a geographical perspective, care should be taken in replacing the geo-part of the query since previous research has shown that automatically reformulated geoqueries are deemed to be irrelevant when the geo-part is changed (Jones et al., 2008).

\subsubsection{Fine-grained classification of geo-modification}

Although the above analysis provides a global view at the syntax level, we would like to understand the semantics of reformulations. By utilizing Rieh \& Xie's (2006) three main facets: content, format, and resource, we were able to zoom in each reformulation action and label it accordingly. The results show that the vast majority of geo-rewritings affect the content of the query more than the other 
two factors. Specifically, $83.2 \%$ of reformulation was made on the content compared with $12.7 \%$ for the format modification. These figures are similar to the generic findings published in Rieh \& Xie (2006).

Under half of those who reformulated their geo-query content viewed at least one result (46.9\%), while $36.7 \%$ of format modification resulted in a click. The highest click percentage was for resource modifications (57.4\%); this was expected to be higher than the others since nearly all of its rewritings are for maps and images of popular places, in which case users' intent is determined and thus the search engine could retrieve highly relevant resources. However, in terms of the content and format, these figures do not seem to be encouraging and give an indication that most users' modifications may not be successful as measured by click data. In general, users' inability to successfully formulate their queries has long been discussed in the IR literature (Gauch \& Smith, 1991) and providing this result; it does appear that such a problem still exists, at least from a geographical perspective.

The 471 geographic sessions were then examined for modification; the results were as follows:

- Searchers were found to modify the geo content of their queries almost equally as they do with the non-geo component: $34.0 \%$ of geo content modification vs. $35.5 \%$ non-geo. In contrast, they seemed to change the format of the geo part less than (30.8\%) the non-geo part (57.3\%). However, based on the fact that the vast majority of modifications were on the content of the query, these results could partially reject the general claim that "when people search for local information or services, they are often fairly confident about the appropriate geo terms. Thus, when users modify their queries, they more often modify the non-geo terms" (Gan et al., 2008: 56). In addition, Jones et al. (2008) found that about $10 \%$ of users change the geographical part of the query. Their study sample, however, is relatively smaller compared with the one used in this study (nearly one order of magnitude difference) may have affected their finding as we found that about $34 \%$ of users modify the geo-part of the query.

- Among geo-content modifications, nearly half the users specialized their geographic references compared to $25.4 \%$ for generalization and $24.8 \%$ for changing the search location.

- The percentage of modifications that changed the search location was 7.0\%. If we include cases where location was either specialized or generalized, the modification figure increased to $8.6 \%$.

- Nearly one in four modifications represent an interruption of either starting a new search or returning to a previously submitted query. The highest percentage of interruptions was from re-visits to previous queries (34.6\%). When we investigated the click stream of those who returned, we found that only $21.2 \%$ of them had clicked on a result. Such a percentage leads to questions over the reasons for this behavior.

Tables 3, 4 and 5 summarize the findings of this stage.

The number of return queries with a lower percentage of clicks was unexpected. It seems that users, after a number of modifications on their geographic query, 
decide to go back to a previously submitted query. This action might be a result of a cognitive process in which the user realizes that a previous query is most relevant to his/her information need or perhaps it could be an indicator of failure in their successive reformulated requests. Another possible interpretation, given the percentage of clicks they made, is that they may have seen an interesting snippet to re-examine. Regardless of what made them perform such an action, this result certainly supports the claim that appropriate caching would enhance the search process (Bruza \& Dennis, 1997).

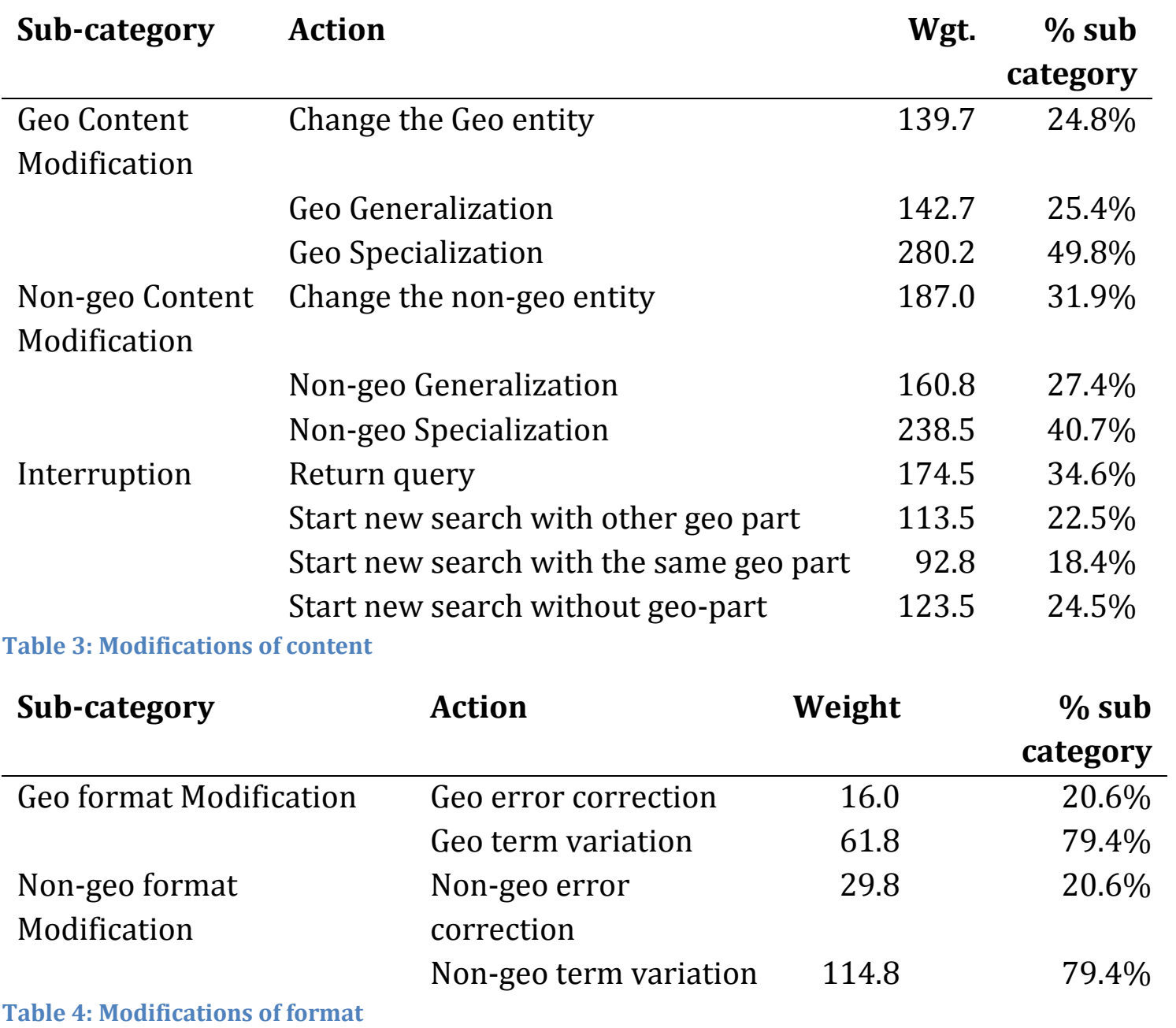

\begin{tabular}{lrrr} 
Action & Weight & \% sub category & \% facet \\
\hline Domain Suffix & 9.0 & $29.5 \%$ & $29.5 \%$ \\
Type of Resource & 21.5 & $70.5 \%$ & $70.5 \%$ \\
Table 5: Modifications of resource & & &
\end{tabular}




\begin{tabular}{|c|c|c|c|c|c|c|c|c|c|c|c|c|c|c|c|c|}
\hline \multirow{2}{*}{ Fro } & \multirow{2}{*}{ To } & \multicolumn{2}{|c|}{ Generalisation } & \multicolumn{2}{|c|}{ Specialisation } & \multicolumn{2}{|c|}{ Change } & \multirow{2}{*}{$\begin{array}{r}\text { New search } \\
\text { (Same } \\
\text { place) } \\
\%\end{array}$} & \multirow{2}{*}{$\begin{array}{r}\text { New } \\
\text { search } \\
(\text { Other } \\
\text { place }) \\
\%\end{array}$} & \multirow{2}{*}{$\begin{array}{r}\text { New } \\
\text { search } \\
\text { (Without } \\
\text { place) } \\
\% \\
\end{array}$} & \multirow{2}{*}{$\begin{array}{r}\text { Return } \\
\text { query } \\
\%\end{array}$} & \multicolumn{2}{|c|}{ Error correction } & \multicolumn{2}{|c|}{ Term variation } & \multirow{2}{*}{$\begin{array}{r}\text { Operato } \\
\text { r usage } \\
\%\end{array}$} \\
\hline & & $\begin{array}{r}\text { Geo } \\
\%\end{array}$ & $\begin{array}{r}\text { Non-geo } \\
\%\end{array}$ & $\begin{array}{r}\text { Geo } \\
\%\end{array}$ & $\begin{array}{r}\text { Non-geo } \\
\%\end{array}$ & $\begin{array}{r}\text { Geo } \\
\%\end{array}$ & $\begin{array}{r}\text { Non-geo } \\
\%\end{array}$ & & & & & $\begin{array}{r}\text { Geo } \\
\%\end{array}$ & $\begin{array}{r}\text { Non- } \\
\text { geo } \\
\%\end{array}$ & $\begin{array}{r}\text { Geo } \\
\%\end{array}$ & $\begin{array}{r}\text { Non- } \\
\text { geo } \\
\%\end{array}$ & \\
\hline \multicolumn{2}{|l|}{ Initial query } & 8.0 & 6.4 & 19.1 & 14.9 & 7.5 & 7.9 & 3.5 & 8.8 & 5.7 & - & - & - & 4.3 & 6.9 & - \\
\hline \multirow[t]{2}{*}{ Generalisation } & Geo & 5.8 & 11.9 & 24.1 & 8.4 & 2.9 & 12.9 & 3.9 & 3.2 & 8.4 & 5.2 & - & - & - & 6.3 & - \\
\hline & Non-geo & 7.8 & 8.4 & 13.7 & 27.4 & 7.0 & 2.7 & - & 3.2 & 4.4 & 8.2 & - & - & 4.0 & 2.9 & 3.9 \\
\hline \multirow[t]{2}{*}{ Specialisation } & Geo & 10.7 & 12.1 & 9.5 & 10.8 & 5.2 & 7.7 & 5.6 & 4.9 & 6.5 & 11.2 & - & - & 3.5 & 6.7 & - \\
\hline & Non-geo & 7.7 & 16.5 & 11.7 & 6.4 & 2.8 & 12.9 & 3.6 & 5.6 & 6.4 & 7.3 & - & 3 & 3.6 & 9.9 & - \\
\hline \multirow[t]{2}{*}{ Change } & Geo & 7.0 & - & 7.4 & 11.9 & 45.3 & 2.9 & 2.5 & 5.8 & 3.3 & 4.1 & 2.5 & - & - & - & - \\
\hline & Non-geo & 6.6 & 6.9 & 14.6 & 10.0 & 4.7 & 26.5 & 2.4 & 3.6 & 4.2 & 8.3 & - & 2.4 & - & 4.8 & 2.3 \\
\hline \multicolumn{2}{|c|}{ New search (Same place) } & 3.8 & 11.5 & 4.9 & 16.1 & 2.5 & 17.8 & 18.0 & 3.3 & 11.5 & 4.9 & - & - & - & - & - \\
\hline \multicolumn{2}{|c|}{ New search (Other place) } & 9.8 & 5.2 & 5.2 & 14.9 & 6.7 & 7.3 & 4.6 & 14.9 & 9.2 & 3.5 & 2.3 & - & 5.0 & 4.0 & \\
\hline \multicolumn{2}{|c|}{ New search (Without place) } & - & 2.0 & 32.3 & 9.1 & - & 4.6 & 12.1 & 6.1 & 17.2 & 5.1 & - & 3.0 & - & 6.1 & - \\
\hline \multicolumn{2}{|l|}{ Return query } & 3.9 & 6.6 & 11.9 & 7.7 & - & 7.3 & 5.2 & 3.9 & 4.9 & 39.5 & - & - & 2.1 & 2.5 & - \\
\hline \multirow[t]{2}{*}{ Error correction } & Geo & 3.6 & 3.6 & 10.7 & 17.9 & 35.7 & & & 7.1 & - & - & 7.1 & 7.1 & 7.1 & & \\
\hline & Non-geo & 8.7 & 8.0 & 15.2 & 4.4 & 2.2 & 2.2 & 8.7 & 8.7 & 4.4 & 4.4 & - & 3.6 & 5.8 & 17.4 & 2.2 \\
\hline \multirow[t]{2}{*}{ Term variation } & Geo & 14.6 & 10.5 & 6.4 & 10.8 & 6.2 & 10.8 & 4.6 & - & 4.6 & 15.4 & 3.1 & - & 8.0 & - & - \\
\hline & Non-geo & 6.5 & 6.5 & 10.8 & 5.9 & 3.8 & 10.8 & 4.3 & 7.5 & 5.4 & 16.1 & - & - & 6.5 & 11.8 & \\
\hline \multicolumn{2}{|l|}{ Operator usage } & 4.7 & 15.6 & 10.9 & 14.1 & - & 9.4 & 3.1 & - & 6.3 & 6.3 & - & - & - & 9.4 & 15.6 \\
\hline
\end{tabular}

Table 6: Transition between different modification actions

\section{Table description:}

- Rows represent the state from which the user is moving (current state) and columns represent "the next expected state".

- Values in bold means the highest probability.

- Resource actions were eliminated from the table given their insignificant role in geo-modification and thus it shall be noted that the values for each row do not amount to $100 \%$ but all of them weight for over $94 \%$. 


\subsection{Actions in geo-reformulation}

Exploring the connection between two successive queries is important either for drawing a complete picture of a user's modification behavior or for developing reformulation tools that can exploit this knowledge in predicting the user's next move. To do this, we adopted the notation of a transition matrix, including in the analysis only the transitions between the content and format facets and ignoring resource as it is negligible in geo-modification. Table 6 shows the detailed findings as laid out in the transition matrix. From the analysis we observed that:

- $73.6 \%$ of users who generalized their queries were likely to perform a specialization on their successive query.

- Users tend to focus their modifications on either the geo or non-geo part of the query in their successive attempts.

- Nearly one in three users who interrupted the sequence of their queries, by searching for a different information need without specifying the location of interest, followed this by determining the location that they wanted to search in. Most of these specialization cases were found to be about the geographic place that the user was searching before the interruption.

- It is evident that when users traced back a query from their search sequence, they were likely to continue in doing this as $39.5 \%$ of "return query" modification actions were followed by the same behavior.

\subsubsection{Analysis of correlated modifications actions}

The aim of this analysis was to investigate the complexity of modification actions that users simultaneously performed on their geographic query. The results showed that only $17.11 \%$ of reformulated queries were having two or three parallel modifications. When we investigated this behavior, the commonest pair of actions was found to have an inverse relationship. In such double modifications, if users generalized the geo-part of the query, they tended to specialize the non-geo component and vice versa. For instance, one of the users made the query "century furniture" and then modified it to "North Carolina furniture manufacturers". However, given the fact that the vast majority of queries were modified using a single action at each time, a reasonable conclusion to be drawn from this is that searchers usually rewrite their geographic information needs in a simple way by performing a single modification action on one of the query components.

\subsubsection{Analysis of Temporal aspects of modifications actions}

It was found that the likelihood of modifying a geographic query was at its peak in the first minute after submission. This illustrates that most users reformulated their requests within seconds although there were occurrences for noticeable amount of modifications after the first minute. A significant amount of the georewriting actions that do not alter the information need happen in this opening time span. In other words, users do not seem to start a new search or change the geo or the non-geo components of their queries as often as the other actions in the initial 60 seconds. This finding is consistent with Lau \& Horvitz's (1999) figures. 


\subsubsection{Actions patterns}

This analysis aims to examine whether users follow a common pattern of modifications in their geo-queries. It was found that the majority of users modified their geo-sessions in quite diverse ways: 225 sessions out of the 471 followed a unique pattern. One of the common patterns occurred when users interrupted their search just after submitting the initial query by starting a new one. Some might argue that this indicates a failure in defining the session boundaries since a session ideally consists of a single information need. On the other hand, the inclusion of such behavior might reveal interesting facts about users' searching activities, especially when the time-frame between the two queries is short.

\subsection{Tasks in geographic query reformulation}

The geo-sessions were analyzed for the purpose of identifying trends in geographic rewritings. This was achieved through a manual inspection of the query sequence within each search session. As a result, four different patterns emerged which are presented in Table 7.

\begin{tabular}{lrr} 
Trend & Frequency & $\mathbf{\%}$ \\
\hline Normal & 318 & $67.5 \%$ \\
Multi-needs & 62 & $13.2 \%$ \\
Multi-places & 43 & $9.1 \%$ \\
Hybrid & 35 & $7.4 \%$ \\
Unclassifiable & 13 & $2.8 \%$ \\
Total & $\mathbf{4 7 1}$ & $\mathbf{1 0 0 . 0 \%}$
\end{tabular}

- Normal approach: This task refers to those sessions in which the user is looking for a single information need in one location (i.e. classical session). The dominant modifications in these queries were found to be generalizations and specializations for both the geo and the non-geo component.

- Multi-needs approach: These were cases in which the user searches for different information needs but within the same location. Users following this approach are likely to have geographically-constrained search tasks such as travel planning or exploratory search. This category shows that not all users reformulate their geographic query either because of inaccurate formulation of the initial query or irrelevant retrieved documents by the search engine. Examples of this category are presented below:

- "ks travel $\rightarrow$ may events in ks $\rightarrow$ ks farms $\rightarrow$ ks farms to visit $\rightarrow$ ks bed and breakfast".(1)

- "Corsica $\rightarrow$ map of Corsica $\rightarrow$ flag of Corsica $\rightarrow$ dress style of Corsica".

- "Wells Fargo $\rightarrow$ Wells Fargo Dallas Locations $\rightarrow$ Forest Cove Apartments Dallas"(2)

- Multi-places approach: refers to those cases when the user searches for a single information need in multiple places. Such behavior might be attributed to users comparing a service in two or more places or perhaps a willingness 
to travel to a nearby location in cases when they could not locate their need in the original one. The following examples illustrate this point:

- "Mortgage Lending $\rightarrow$ Mortgage Lending Herndon $\rightarrow$ Mortgage Lending Missoula".

- "ford of Grapevine $\rightarrow$ ford of Fort Worth $\rightarrow$ ford of Richardson $\rightarrow$ ford of Plano $\rightarrow$ ford of Duncanville"

- Hybrid approach: refers to the situation in which the user mixes between two of the former approaches in one session. An example of this is presented below:

- $\quad$ "Pennsylvania personal license plates $\rightarrow$ penna personal license plates $\rightarrow$ penna personal plates $\rightarrow$ New York marathon $\rightarrow$ New Jersey marathon".

- "Charlotte fire department $\rightarrow$ Rock Hill fire department $\rightarrow$ Rock Hill,sc fire department $\rightarrow$ US LEC $\rightarrow$ US LEC Charlotte, nc".

\section{Discussion}

IR systems are traditionally designed to retrieve results in a stateless contextunaware manner. They rarely make use of a richer context of users' interaction with the system in a systematic way that approximates users' intents, topical interests and possible future actions. Reformulation studies provide the opportunity to analyze richer behavioral signals perhaps reflecting a richer picture of users' needs. However, it is usually difficult to provide global explanations for the reasons that lead users to reformulate their queries. In this study we focused on an important sub-type of search queries and systematically analyzed its reformulation characteristics. Unlike previous studies in the geographical queries domain, we focused on sessions as the unit of analysis and not only pair of queries.

The initial findings in regard to the mean session length and the proportion of geographical queries in the query log are consistent with previous findings by Silverstein et al. (1998) and Sanderson \& Kohler (2004). It is interesting to note the static behavioral side of this finding, particularly in the context of geo queries, given the fact that users' interest in geographical queries remained at a constant level compared with the nearly five years older dataset used in Sanderson \& Kohler (2004). This coincides with the claim made by Spink et al. (2001: 227) that "The Internet changes fast. In contrast, people, their information needs, and behavior do not". However, it would also be interesting to examine these findings again after the wide adoption of smart phones and tablets which may have increased people interests in searching for geographically constrained information.

In regards to the modification actions, we found that a significant percentage of the initial modifications were associated with the geographical part of the query. This might have been caused by the fact that the geographical part of the query is usually not part of the information need but has a disambiguation function. In their subsequent actions, users tendency to focus their reformulation actions on a single part of the query (geo or non-geo) by oscillating between generalization and specialization indicates that they are mostly uncertain about their keywords 
choices and engage in an interactive cycle of querying, examining and refining. In addition, the finding that users who interrupted the initial query by starting a new search usually reformulate the new query by adding the same geographical entity used in their initial query. This suggests that using geographical entities that are used during the session a short-term indicator of context might help in personalizing the results for new queries that occur within a short-time window.

The analysis of the search tasks revealed that not all session were about a single information need (about 67\% all sessions) but users were also searching for multi-need in one place or a single information need in multi-places. This consideration of sessions' characteristics would probably have implications on designing various IR applications. For example in the context of session-based retrieval, it would desirable to utilize such prior knowledge to either explore and present relevant but novel needs for those believed to belong to the multi-need category or to present results from different geographical entities for those belonging to the multi-places category. Similar information could be utilized for advertisements as well as diversification.

However, these results are limited in a number of ways. First, the data was collected during July 2006, which might mean an increase in the number of geographical interests during the summer holiday. Secondly, although this dataset is an order of magnitude larger than the previous geo reformulation findings reported in Jones et al. (2008), it would still be interesting to scale the experiment to automatically classifying a larger datasets.

In addition, this paper inherits some of the limitations of unobtrusive data collection approaches, i.e. query log analysis. Search, particularly in the context of multi query sessions, is an interactional process where each party's actions affect the other. This means that the search engine might have taken the current location of the user into account at retrieval time, which would affect users' reformulation behavior. However, our work focuses on behavioral analysis of users' geographical needs that span at least three subsequent queries and the mechanisms by which users searched rather than on how to use location signals in scoring relevant information. Sessions containing three or more queries suggest untypical behavior compared with those having a single query and are more likely to induce patterns and findings that have the potential to guide location personalization or query suggestions methods. In other words, adapting the search engine to a particular view of users' needs, i.e. location personalization, requires an understanding of their search behavior to which this paper contributes.

Moreover, while this study uses a dataset collected at a time where location personalization was less prominent than it is now with commercial search engines, we believe that this might have the advantage of controlling the experiment by eliminating actions taken by the search engine that could have suppressed users' behavior with respect to the study goals. In general, it would be of interest to conduct further studies in controlled settings to investigate the effect of current location personalization approaches on users' search behavior , with reference to this study findings, particularly with the widespread usage of 
smart phones and tablets that might have an effect on the proportion of geographical queries as well.

\section{Conclusion}

This paper presents a detailed analysis of geographic search sessions. At a general level, we first re-examined the proportion of reformulation sessions and found that over $38 \%$ of users submitted more than one query during a session. This figure is less than that derived from earlier Excite log studies (Wolfram D. et al., 2001), suggesting a probable modest improvement in the retrieval models. By analyzing the sampled data, the proportion of geographical queries were found to be consistent with that of Sanderson and Kohler (2004), indicating a stable inclination to use geographical entities as query modifiers. In addition, it was also observed that users tend to modify the non-geographical part of the query almost as often as they reformulate the geo part. We also noticed that in one out of four reformulation cases users were interrupting their original search task. The dominant behavior when interruption occurs is either to start another geographically related search task $(40 \%)$ or to return to previously submitted query (34.6\%). Different from previous automated approaches, we then followed a qualitative method to semantically annotate and induce geographic search characteristics. We have shown that about $30 \%$ of reformulations in such sessions are not a result of unsuccessful first attempt; but rather indicating complex search tasks, namely multi-needs in one place or a single-need in multiple places. This suggests that it might be useful to diversify geographicallyrelated results to account for such behavior. We have also provided a probabilistic transition analysis which shows that users tend to oscillate between modification actions (e.g. generalization to specialization and vice versa) while persisting in modifying the geo or non-geo part of the query. A possible future work is to utilize this scheme in automatically classifying a larger collection, especially after the widespread use of smart phones and the likelihood of an ever increasing importance of geographical queries.

\section{References}

Bendersky, M. \& Croft, W.B. (2009)."Analysis of long queries in a large scale search log". Proceedings of the 2009 workshop on Web Search Click Data ACM, Barcelona, Spain, pp. 8-14.

Bendersky, M., Metzler, D., \& Croft, W. B. (2011). Parameterized concept weighting in verbose queries. Proceedings of the 34th international ACM SIGIR conference on Research and development in Information Retrieval, SIGIR '11 (pp. 605-614). New York, NY, USA: ACM.

Broccolo, D., Marcon, L., Nardini, F. M., Perego, R., \& Silvestri, F. (2012). Generating suggestions for queries in the long tail with an inverted index. Information Processing \& Management, 48(2), 326-339.

Broder, A. (2002). "A taxonomy of web search". SIGIR Forum, 36 (2), 3-10.

Bruza, P.D. \& Dennis, S. (1997). "Query-reformulation on the internet: Empirical data and the hyperindex search engine". Proceedings of the RIAO Conference: Intelligent Text and Image Handling, pp.488-499.

Chau, M., Lu, Y., Fang, X. \& Yang,C.C. (2009). "Processing and Analysis of Search Query Logs in Chinese”. In: Jansen,B.J., Spink,A., Taksa,I. (ed.), Handbook of Research on Web Log Analysis, pp. 378-388. London: IGI Global. 
Cucerzan, S. \& White, R.W. (2007)." Query suggestion based on user landing pages". Proceedings of the 30th annual international ACM SIGIR conference on Research and development in information retrieval ACM, Amsterdam, The Netherlands, pp. 875-876.

Cui, H., Wen, J.R., Nie, J.Y. \& Ma, W.Y. (2003). "Query expansion by mining user logs". Knowledge and Data Engineering, IEEE Transactions on, 15 (4), 829839.

Efthimiadis, E.N. (2008)." How do Greeks search the web?: a query log analysis study". Proceeding of the 2nd ACM workshop on Improving non english web searching ACM, Napa Valley, California, USA, pp. 81-84.

Gan, Q., Attenberg, J., Markowetz, A. \& Suel, T. (2008)."Analysis of geographic queries in a search engine log". Proceedings of the first international workshop on Location and the webACM, Beijing, China, pp. 49-56.

Gauch, S. \& Smith, J.B. (1991). "Search improvement via automatic query reformulation". ACM Trans. Inf. Syst., 9 (3), 249-280.

Jansen, B.J., Spink, A., Bateman, J. \& Saracevic, T. (1998). "Real life information retrieval: A study of user queries on the web". SIGIR Forum (ACM Special Interest Group on Information Retrieval), 32 (1), 5-17.

Jansen, B.J., Spink, A. \& Saracevic, T. (2000). "Real life, real users, and real needs: A study and analysis of user queries on the Web". Information Processing and Management, 36 (2), 207-227.

Jansen, B.J. \& Pooch, U. (2001). "A Review of Web Searching Studies and a Framework for Future Research". Journal of the American Society for Information Science and Technology, 52 (3), 235-246.

Jansen, B.J. \& Spink, A. (2006). "How are we searching the World Wide Web? A comparison of nine search engine transaction logs". Information Processing \& Management, 42 (1), 248-263.

Jansen, B.J., Booth, D.L. \& Spink, A. (2009a). "Patterns of query reformulation during Web searching". Journal of the American Society for Information Science and Technology, 60 (7), 1358-1371.

Jansen, B.J., Spink, A. \& Taksa, I. (2009b). "Research and Methodological Foundation of Transaction Log Analysis". In: Jansen,B.J., Spink,A., Taksa,I. (ed.), Handbook of Research on Web Log Analysis, pp. 1-16. London: IGI Global.

Jones, R., Rey, B., Madani, O. \& Greiner, W. (2006). "Generating query substitutions". Proceedings of the 15th International Conference on World Wide Web, pp. 387-396.

Jones, R., Zhang, W.V., Rey, B., Jhala, P. \& Stipp, E. (2008). "Geographic intention and modification in web search". International Journal of Geographical Information Science, 22 (3), 229-246.

Kohler, J. (2003). Analysing search engine queries for the use of geographic terms. MSc, University of Sheffield.

Lau, T. \& Horvitz, E. (1999). "Patterns of search: Analyzing and modeling web query refinement". Proceedings of the 7 th International Conference on User Modeling, pp. 119-128.

Liu, Y.H. \& Belkin, N.J. (2008). "Query reformulation, search performance and term suggestion devices in question-answering tasks". Proceedings of the second international symposium on Information interaction in context, London, United Kingdom, pp. 21-26. 
Martins, B., Silva, M.J., Freitas, S. \& Afonso, A.P. (2006). "Handling Location in Search Engine Queries". GIR'06: Proceedings of the 3rd Workshop on Geographical [Online].

http://www.geo.uzh.ch/ rsp/gir06/papers/individual/martins.pdf [Accessed 25 June 2009]

Park, S., Lee, J.H. \& Bae, H.J. (2005). "End user searching: A Web log analysis of NAVER, a Korean Web search engine". Library and Information Science Research, 27 (2), 203-221.

Pass, G., Chowdhury, A. \& Torgeson, C. (2006)." A picture of search". Proceedings of the 1st international conference on Scalable information systems ACM, Hong Kong [Online].

Rieh, S.Y. \& Xie, H. (2006). "Analysis of multiple query reformulations on the web: The interactive information retrieval context". Information Processing and Management, 42 (3), 751-768.

Rose, D.E. \& Levinson, D. (2004). "Understanding user goals in Web search". Proceedings of the 13th international conference on World Wide Web, New York, USA, pp. 13-19.

Ross, N.C.M. \& Wolfram, D. (2000). "End user searching on the Internet: An analysis of term pair topics submitted to the Excite search engine". Journal of the American Society for Information Science and Technology, 51 (10), 949958.

Sanderson, M. \& Kohler, J. (2004). "Analyzing geographic queries". Proceedings of Workshop on Geographic Information Retrieval SIGIR [Online]. http://www.geo.uzh.ch/ rsp/gir/abstracts/sanderson.pdf [Accessed 10 September 2012]

Silverstein, C., Henzinger, M., Marais, H. \& Moricz, M. (1998). "Analysis of a very large AltaVista query log". Technical Report [Online]. ftp://gatekeeper.dec.com/pub/dec/SRC/technical-notes/SRC-1998-014.pdf [Accessed 10 September 2012].

Spink, A., Jansen, B.J. \& Ozmultu, H.C. (2000). "Use of query reformulation and relevance feedback by Excite users". Internet Research, 10 (4), 317-328.

Spink, A., Wolfram, D., Jansen, M.B.J. \& Saracevic, T. (2001). "Searching the Web: The Public and Their Queries". Journal of the American Society for Information Science and Technology, 52 (3), 226-234.

Spink, A. \& Ozmultu, H.C. (2002). "Characteristics of question format web queries: An exploratory study". Information Processing and Management, 38 (4), 453-471.

Spink, A., Ozmutlu, S., Ozmutlu, H.C. \& Jansen, J. (2002a). "US versus European Web searching trends". ACM SIGIR Forum, 36 (2).

Spink, A., Jansen, B.J., Wolfram, D. \& Saracevic, T. (2002b). "From E-Sex to ECommerce: Web Search Changes". Computer, 35 (3), 107-109.

Wang, L., Wang, C., Xie, X., Forman, J., Lu, Y., Ma, W.-Y. \& Li, Y. (2005). “Detecting dominant locations from search queries ". Proceedings of the 28th annual international ACM SIGIR conference on Research and development in information retrieval ACM, Salvador, Brazil, pp. 424-431.

Welch, M.J. \& Cho, J. (2008)." Automatically identifying localizable queries" .Proceedings of the 31st annual international ACM SIGIR conference on Research and development in information retrieval ACM, Singapore, Singapore, pp. 507-514. 
Wolfram, D., Spink, A., Jansen, B.J. \& Saracevic, T. (2001). "Vox populi: The public searching of the Web". Journal of the American Society for Information Science and Technology, 52 (12), 1073-1074.

Yi, X., Raghavan, H. \& Leggetter, C. (2009).” Discovering users' specific geo intention in web search ". Proceedings of the 18th international conference on World wide web ACM, Madrid, Spain, pp. 481-490.

Zhang, Y. \& Moffat, A. (2006). "Some observations on user search behavior". Proceedings of the 11th Australasian Document Computing Symposium, pp.18.

Zhu, X., Guo, J., Cheng, X., Du, P., \& Shen, H.-W. (2011). A unified framework for recommending diverse and relevant queries. Proceedings of the 20th international conference on the World Wide Web, WWW'11 (pp. 37-46). New York, NY, USA: ACM. 